\title{
La Universidad Autónoma de Chihuahua en Camino a la Departamentalización; Innovación para Mejorar el Desempeño Académico y Reordenamiento Administrativo
}

The Autonomous University of Chihuahua on the Way to the

Departmentalization; Innovation to Improve Academic

Performance and Administrative Reorganization

Juan de Dios Ramírez Cereceres ${ }^{1}$ y Santiago Pinedo Irigoyen ${ }^{2}$

\section{Resumen}

En este escrito se explica en qué consiste el procedimiento de Departamentalización que se anunció hace algunos meses en la UACH; se expondrán el grado de factibilidad, ventajas o desventajas que se plantean. Se trata de una estrategia de ajuste presupuestario que respalda una correcta redistribución de fondos y mejora administrativa tendiente a optimizar la infraestructura, vinculación social, mejoría de la calidad y profesionalización docente, la investigación y el impulso de los programas educativos; o una política de reacción ante los recortes de partidas federales que afectan concretamente al sector educativo y después áreas laborales de la institución.

Palabras Clave: Departamentalización, gestión administrativa, vinculación universitaria, innovación, docencia.

\begin{abstract}
This work describes the procedure of departmentalization that was announced a few months ago in the UACH; will the degree of feasibility, advantages or disadvantages that arise. This is a budgetary adjustment strategy that supports a correct redistribution of funds and administrative improvement aimed to optimize infrastructure, social bonding, improvement of the quality and professionalization of teaching, research and the momentum of educational programs; or a policy of reacting to cutbacks in federal items that affect specifically the education sector and after labor areas of the institution.

\footnotetext{
1 Profesor de tiempo completo en la Facultad de Ciencias Políticas y Sociales de la Universidad Autónoma de Chihuahua, jdramirez@uach.mx

2 Profesor de asignatura de la Facultad de Ciencias Políticas y Sociales de la Universidad Auónoma de Chihuahua.spinedo@uach.mx
} 
Keywords. Departmentalization, administrative management, linking university education, innovation, teaching.

\section{Introducción}

Del latín universitas, el concepto Universidad se define como un organismo de enseñanza superior que comprende varias facultades y que confiere los grados académicos correspondientes. Según las épocas y países puede comprender colegios, institutos, departamentos, centros de investigación, escuelas etc, (RAE, 2014).

Colman cita a Ulpiano al referirse a la Universidad como una concepción formada por todo un grupo de trabajadores, pues cada uno desde cierto punto de vista individual o conjunto forman o hacen universitas (Colman, 2000).

Mientras tanto para Marciano, citado por Jovellanos comenta que la Universidad puede ser comparada con los estadios de las ciudades, pues todos pueden tener acceso a ella y quienes lo hacen pasan a integrar un cuerpo o grupo específico que puede ser diferenciables de los demás. Cicerón por su parte relaciona el universitas rerum como la totalidad de las cosas que integran el universo y universitas generis humani igual a la suma de las personas en el espacio y tiempo que constituyen la humanidad (Jovellanos, 2017).

Tras comprender el término, se define que se trata de una de las estructuras educativas más antiguas en la historia del hombre y que a pesar de todo ha conservado su esencia a través del tiempo, incluso desde que se instituyó que habría de ser un centro formativo sólo para hombres, a cargo de la iglesia en la era del medioevo con una capacitación totalmente apegada a la formación religiosa y que luego se abrió al mundo entero.

Las circunstancias históricas favorecieron la aparición de las primeras universidades y posteriores métodos de enseñanza sobre planteamientos más laicos. Después, ante su necesaria transición hacia una estructura organizativa encaminada a dar respuesta a los problemas resultado del crecimiento y apertura de la formación superior a través del tiempo, sobre todo en el siglo $\mathrm{XX}$, vieron la luz arquetipos del desarrollo organizativo y operación de centros educativos,

Uno de ellos fue el Modelo Napoleónico, de organización tradicional y 


\section{DOXA}

pISSN: $2395-8758$

eISSN: en trámite

enfocado a la educación comparativa que implicaba la visualización analítica de la estructura actual. Este diseño ponderó la formación profesional, la integración de Facultades y la docencia de grado, además de otorgar licencias para ejercer legítimamente la profesión, contribución a la sociedad y propuestas de solución a problemas prácticos (Tünnermann, 2003).

Posteriormente, con el surgimiento de los institutos técnicos, tecnológicos y escuelas normalistas para la formación de maestros, el diseño del patrón Napoleónico va siendo superado o se vuelve una opción redundante a comparación de otras instituciones que en poco tiempo se supera al decidir dejar de lado el modo de trabajo acostumbrado que se dedicó a "maquilar" expertos de acuerdo a lo que el estado demandaba en ese contexto histórico. Por otra parte, surge no con mucha diferencia de tiempo, pero si con otra propuesta considerada más completa, el patrón humboldtiano, también conocido como alemán, que se constituyó a través de instituciones con docentes funcionarios y la idea muy marcada de fomentar el conocimiento científico, es decir buscando formar personal con la preparación necesaria para dar solución a los problemas reales que presentaba la sociedad o los mercados laborales no sólo de actualidad sino con prospectivas futuras (Rondón \& Perozo, 2003).

La novedad de este sistema se basa en que puede ser aplicable en todas las áreas, no importando si se trata de una universidad de grandes proporciones o una muy pequeña, con pocos o muchos campus o de reciente o antigua creación, aparentemente es más eficaz.

\section{La Estructura de la Universidad Autónoma de Chihuahua}

La Universidad Autónoma de Chihuahua, según la Ley Orgánica, está constituida por las siguientes dependencias (UACH, 2008):

-Las Unidades, Estructuras Académicas y de Extensión, y las que se establezcan con el propósito de impartir docencia, generar investigación y realizar extensión y difusión de la cultura, en los términos y especificaciones de las disposiciones reglamentarias y de las políticas académicas universitarias que correspondan;

-La Administración Central, integrada por la Rectoría, Direcciones, Coordinaciones, Departamentos, Consejos y organismos que se estimen necesarios". 
El Artículo Séptimo hace referencia a los órganos de gobierno internos que abarcan:

-El Consejo Universitario.

-El Rector.

-Los Consejos Técnicos de las Unidades Académicas.

-En general quienes, conforme a esta Ley y sus Reglamentos, tengan facultades de decisión en sus respectivas áreas.

La estructura de la Máxima Casa de Estudios del Estado de Chihuahua, puede ser considerada funcional, pues sigue siendo la más importante formadora de profesionistas, es influyente en los ámbitos político, social y económico del estado y se preocupa por crecer a la par la transformación tecnológica.

De cualquier manera, se han realizado intentos variados por ir avanzando con reformas de mejoramiento y adelanto en los sectores docentes, educativos y de investigación (Muñoz, 2017).

Pero, a pesar del esfuerzo significativo, el formato bajo el cual se rige esta institución, sigue considerándose rígido en la estructura curricular y de claras tendencias profesionalizantes, que si bien ha buscado enlazar el trabajo investigativo con la realidad social, no se dirige por completo a propiciar una vinculación estrecha con la sociedad para responder a problemas añejos con efectos permanentes.

Por ello no es extraño que a finales de los años 60's varios expertos externos recomienden que si la Universidad más importante de Chihuahua trabaja en parte bajo el esquema del diseño francés, que aparentemente sirvió de fundamento para la formación de instituciones de educación superior; valdría la pena buscar una propuesta basada en el Modelo humboldtiano para determinar la factibilidad de aplicación en la Universidad.

Durante la presentación del primer informe de actividades del Rector de la UACH se anunció que en la primera parte de su administración se irá tras la renovación universitaria, que no es más que el reemplazo de un modelo para practicar el funcionamiento humboldtiano, lo que no necesariamente debe ser una nueva reestructura, pero aclarando que con el tiempo si se llegará a ella (Fierro, 2017).

Las reacciones no se hicieron esperar, pues la percepción inicial, seguramente a causa del desconocimiento sobre la real efectividad de este sistema matricial, hizo pensar de inmediato que las medidas iniciales se enfocarían a contener los egresos provocados por una abultada nómina. 


\section{DOXA}

pISSN: 2395-8758

eISSN: en trámite

Por lo que tal escena vislumbró reducción departamental y, por lo tanto de personal; separación de funciones y de autoridad por parte de los directores de las facultades, al igual que el correspondiente efecto en cada estructura de las unidades académicas; sin embargo una vez explicado se puede suponer que el resultado paulatino de esto será benéfico para el funcionamiento integral.

Se percibe por parte de los promotores de este plan piloto que, de planearse y efectuarse correctamente, habrá beneficios en los aspectos administrativos, académicos, institucional, organizativo y demás.

Inicialmente habrá que arrancar con una solicitud de reforma a la Ley Orgánica para integrar la propuesta administrativa que anunció el Rector en funciones y si se desarrolla adecuadamente la primera implicancia se reflejaría en un ahorro del $30 \%$ al $40 \%$ en el gasto corriente, prescindiendo de ciertas posiciones que de una $\mathrm{u}$ otra forma realizan actividades paralelas a las que desarrolla la Rectoría.

Es de ahí de donde surge la incertidumbre del personal, aún y cuando no se anunció por parte del directivo que eso significaría el despido de personal:

“...se inicia una renovación universitaria y en este sentido hay que analizar todos las causas, todos los sistemas dentro de la Universidad, desde lo que es el aula, la interacción Maestro-Alumno, las formas de dar la clase.

Eso va a producir un análisis de las carreras, las licenciaturas, las unidades de docencia, de la conformación de las facultades; hay que revisar esa parte. Pero eso es un proceso que viene desde la revisión del aula y en ese sentido desemboca en la revisión de la Legislación; es integral, busca el mejoramiento y reducción en todos sus diseños, no quiere decir que nos vayamos a departamentalizar, pero no quiere decir que tampoco lo vamos a hacer, quiere decir que estamos haciendo un análisis y el resultado precisamente dará opciones sobre lo que se tiene que hacer..." (Fierro Ramírez, 2017).

Posteriormente aclaró la necesidad de la práctica analítica que representa esta estrategia, inicialmente para sondear la estructura académica, así como los alcances en los niveles extra académicos, de investigación, extensión y difusión de la cultura para conjuntar un todo que va a producir un reordenamiento en el mismo sentido para eficientar los recursos, sin definir si con ello se obtendrían mayores recursos, pero si asegurando que los recursos 
existentes pueden distribuirse de mejor manera.

Con lo anterior no desaparecerían las 15 facultades existentes, más bien con la aprobación del Consejo Universitario para realizar un ajuste a la Ley Orgánica y con la ayuda de especialistas que han participado en otros centros concretando este esfuerzo multidisciplinar, se prevé se organice en forma similar a instituciones de educación superior en Europa, Estados Unidos, México e incluso la UACJ que desde hace una década decidió experimentar con el modelo, destacando uno de los puntos torales de este sistema, que es la de albergar diversas carreras con similar perfil en institutos en Ciencias Sociales, Ciencias Exactas, Ingenierías y de la Salud.

\section{Sobre el Modelo Alemán.}

El desarrollo del Modelo Humboldtiano, también llamado Matricial se debe a la propuesta del lingüista Wilhelm von Humboldt quien al final de los años 1800 's influyó notoriamente en el mejoramiento administrativo, llegando a ser conocido como el iniciador de la Revolución Escolar al promover la sustitución de un sistema de educación de enseñanza abierta a todo el mundo para enfatizar en la formación humana por medio de una propuesta pedagógica que fructificara en el diseño de experimentos educativos actuales; de ahí procede la teoría que actualmente conocemos como Humanismo (Rodríguez, 2006).

Plantea que la culminación del género humano no radica de ninguna manera en "la realización de una perfección general abstracta", postura que previamente se había diseñado al sostener que el hombre en el transcurso de su formación necesitaba socializar y por consiguiente comprometerse con el "Todo".

Años después Dietrich Benner consideró que es gracias a las propuestas reformistas y atrevidas para su tiempo por parte de Humboldt, por lo que ahora percibimos una importancia por los aspectos fundamentales de la teoría moderna de la educación, pues nos volvemos más perceptivos sobre la conveniencia de profundizar en la teoría y la práctica (Benner, 1990).

Incluso de su propuesta se deriva lo que ahora es conocido como research university, que es uno de los fundamentos de la relación que existe entre las instituciones superiores y las empresas de donde se ha generado beneficio mutuo por medio de los resultados en la investigación desarrollada $\mathrm{y}$ 


\section{DOXA}

pISSN: 2395-8758

eISSN: en trámite

aplicada, así como en la transferencia de tecnológica de avanzada.

Finalmente, de acuerdo a lo expresado por Leitner, el talante más característico del modelo alemán o Matricial se evidencia en el complemento que existe entre la investigación y la docencia, sobre lo cual señaló Humboldt en 1810, que las instituciones de educación superior o universidades estaban obligadas a plantear la docencia como si ésta constituyera un panorama de problemas comunes que aún no habían sido resueltos y por lo tanto se encontrarían siempre sujetos a avances derivados de investigación (Leitner, 2008), la globalización y los avances tecnológicos que han servido para fortalecer las mejoras educativas solamente en lo que al acceso inmediato a la información se refiere y la necesidad de cumplir con la función social para que la que fueron creadas.

Las instituciones educativas de nivel superior han participado desde antaño en esa modernización, sin embargo la especialización de las actividades no son una propuesta reciente, pues efectivamente el ejemplo viene del que tendemos a llamar Primer Mundo, donde en un amplio segmento se han consolidado en cuanto a la estructura universitaria, logrando ser más eficientes con la formación de mejores profesionistas, sin dejar de lado el respeto a los valores individuales y los aportes sociales derivados de la investigación.

Está implícito el diseño de estrategias administrativas que se diseñan o actualizan cada cierto periodo en las instituciones educativas a fin de mejorar de manera significativa la gestión, pero más allá de eso se vuelve un programa integral que incluye la mejora continua, la generación de satisfacción del docente, los diseños programáticos de las carreras y con la certidumbre de que el resultado se vea reflejado en la entrega a la sociedad de una cantidad de profesionistas que se especializan en su área, comprometidos con la solución de la problemática social y con un alto grado de formación en valores (Tisnado, 2013).

En ese contexto, la propuesta de Arroyo Vázquez considera a las universidades como agentes clave para que arranque y se consolide un proyecto de Escuela Emprendedora desarrollando al mismo tiempo actividades de educación e investigación, así como el fomento a la cultura al interior de la misma; una contribución al desarrollo económico y social (Vázquez, 2016).

Es esa aparentemente la visión del Rector de la Autónoma de Chihuahua, 
Luis Alberto Fierro Ramírez, quien vislumbra la consolidación de un proyecto progresivo e integral en la Alma Mater, de una gran cantidad de chihuahuenses y mexicanos por este medio para el avance sustantivo en el diseño educativo en el que se lleva varios años trabajando, desde la modificación de la currícula en los programas, hasta las estrategias para el desarrollo interno que incluye las finanzas y el personal docente.

Por eso, hablar de la diversificación de funciones en la UACH, es referirse a un esquema que habrá de llevarse paso a paso y es probable que ni siquiera se alcance a concretar en la actual administración; a pesar de que Fierro Ramírez anunció que durante todo el año 2018 se trabajaría en el análisis de la estructura académica, administrativa, de planeación, extensión, coordinaciones y áreas de apoyo para obtener un esquema real del estado en que se encuentra y fijar las posibilidades de departamentalizar.

\section{Similitudes y Diferencias entre Modelos}

El cuadro comparativo que se nos ofrece a continuación, nos muestra las similitudes y diferencias entre cada uno de los modelos con los que aún se trabaja en muchas universidades de México; por un lado lo propuesto en la era Napoleónica para dar una estructura coherente y social al movimiento de formación superior, y por el otro el que, si bien tampoco es muy nuevo, si fue concebido desde el inicio para ser partícipe del constante movimiento evolutivo en la preparación universitaria.

El modelo Humboldtiano con un aspecto más humanista para concretar un enlace más fortalecido con la sociedad de esa época, pero apostando a que la población aumentaría, las ideas sufrirían transformaciones impensables, por lo que tuvo un formato se puede decir auto ajustable a la dinámica social a través de los siglos. 


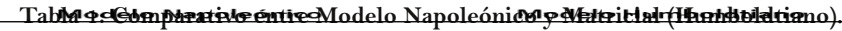

\begin{tabular}{ll}
\hline El Desarrollo Organizacional se & El Desarrollo Organizacional se basa \\
basa en los intereses de quienes & en las relaciones humanas, el \\
ejercen el poder. & compromiso individual para "aceptar" \\
& los objetivos organizacionales. \\
El nivel en la dimensión vertical & Las tres bases al departamentalizar \\
determina la autoridad $y$ pader, e & son: \\
influencia, privilegio, status $y$ & Función, Programas, Localización.
\end{tabular}

recompensas.

Relativamente cerrada, intentos por seleccionar $\checkmark$ minimizar llas fuerzas ambientales y reducir la incertidumbre

Útil para pequenas organizaciones $\checkmark$ la disciplina es fácil de mantener es rígida e inflexible.

Centralizada, concentrada hacia los niveles altos, tiende a estar relativamente fija.

Los niveles jerárquicos son el conjunto de órganos agrupados de acuerdo al grado de autoridad $x$ responsabilidad independientemente de la función que realicen.

La organización depende de hombres clave, lo que acasiona que no se dediquen a labores directivas sino de operación simplemente. La diferenciación se da por pugnas competitivas. Mavor facilidad para la toma de decisiones $y$ en su ejecución. No hay conflictos de autoridad ni fuga de responsabilidad.

Tendencia a la competencia entre el poder central Y las subsistemas. (Fuente de Problemas).

El control se logra por la supervisión permanente.

Se establece la Jerarquía $\vee$ burocracia pérdida de tiempo al comunicar una orden.

Tendencia a extralimitar sus alcances, a la diferenciación, integración, perdiéndose la perspectiva de un solo sistema; sólo es garantía la adecuada distribución de la autoridad. Atribuida a puestas específicas $\gamma$ roles.

Mayor diferencia entre las niveles. La estructura no permite atender necesidades.

Relativamente abierta, diseñada para adaptarse a las

influencias del medio y manejar la incertidumbre.

Flexible. Permite que las personas

puedan cambiar

de tarea cuando sea necesario

Descentralizada de toda la

organización.

Continuamente adaptándose a nuevas situaciones.

El reagrupamiento según la función se logra mediante una división orgánica que permite a la organización desempeñar con eficiencia sus diversas actividades.

Tendencia a la diferenciación, y a la integración

sólo es garantía a la adecuada distribución de la

autoridad Matricial

Coordina la satisfacción de necesidades para mejorar el praducto, para satisfacer el programa Y para atender sus integrantes. Facilita la solución de las problemas que surgen de la diwisión del trabaja. Estimula la cooperación y evita las conflictos laborales.

Funciona a través de reuniones. La responsabilidad es tarea de tados. Propicia una comunicación interdepartamental. Priva la democratización. Nivel de organización horizontal, no permite la buracracia.

En esta evolución se da una red de interacciones de tipo horizontal, son relaciones de trabajo o tareas independientes que sefralan los roles individuales de acuerdo a las objetivas organizacionales. La estructura no permite atender necesidades.

Lo importante es la calidad, pertinencia $y$ el sentida de pertenencia.

Fuente: Gaceta Universitaria. Reglamento de la Academia de Zulia (2007).

Vol. 8 Núm. 14 Enero - Junio 2018 
Es visible que la comparación de planteamientos, si bien exhibe similitudes como la prevalencia de conceptos de autoridad, jerarquía y demás; también se presenta una diversidad de criterios sobre la estructura organizativa, mientras que el estándar Napoleónico no ha presentado ajustes significativos y si se ha producido alguno ha sido solamente en cuanto al nombre; el Matricial permite entre otras cosas, la creación de programas finitos y de matrícula controlada.

\section{En qué Consiste la Departamentalización.}

Se considera un avance administrativo y de gestión inteligente. En las instituciones de Educación Superior es vista como una herramienta de importancia en lo que se refiere a los proyectos de organización universitaria en el país, pues frecuentemente va ofreciendo respuesta a los planteamientos de la sociedad contemporánea, a la vez que responde a las demandas para su desarrollo de acuerdo al momento histórico en que se sitúa.

Esto sucede en el periodo en el que la mayoría de los países capitalistas apostó por la división lógica de trabajo, pues era justamente lo que se necesitaba en aquellas sociedades que se encontraban en constitución, fuera por la forma en que organizaban y distribuían el conocimiento o por la dinámica y eficiencia con que ejercían la distribución de sus recursos (Ibarra, 1998).

De cualquier manera en México este tipo de organización no tardó en llegar y a la fecha ya existen varias universidades que trabajan en base a este sistema, pues a decir de muchos, se hace necesaria la renovación universitaria toda vez que el Modelo Napoleónico se ha vuelto obsoleto y la transición es incluso perceptible para los propios estudiantes, que podrían considerar que su preparación durante los años universitarios no bastará para enfrentar las demandas en un mundo globalizado y exigente.

La relevancia que ofrece el Modelo Matricial o de Humboldt es, entre muchas cosas, la garantía de la desburocratización, lo que sin duda habrá de evidenciarse en la pronta respuesta en cualquier trámite administrativo.

Otra es la garantía que le ofrece al futuro profesionista respondiendo al temor que se acaba de mencionar, pues aparentemente garantiza la pertinencia 


\section{DOXA}

pISSN: 2395-8758

eISSN: en trámite

social, ya que su Alma Mater está obligada a desarrollar propuestas dirigidas a dar respuesta a los graves problemas de la sociedad, por medio de aplicación de programas que prioricen la formación humanística, científicotecnológica y puntual en cuanto a la solución de inconvenientes precisos (Rondón \& Perozo, 2003).

Es decir, dejar claro que el hombre es un ente integral que habrá de trascender por sobre la problemática social, y será el producto que genere la institución lo que ofrezca esa solución.

Siendo reiterativos con la estructura matricial, se busca guiar la propuesta para que la educación superior mantenga también una relación cercana con la comunidad a la que se debe y con los poderes del estado o gobierno, pues es ahí donde habrán de ser diferencia los profesionales que la representarán.

Finalmente, con esa sinergia social se puede también imponer una dinámica en la que se integren los miembros de la comunidad para que el final todo el conjunto haga realidad cualquier demanda hecha por el mismo sector.

Ejemplificando, la disposición de las primeras universidades a adoptar el sistema matricial en los Estados Unidos, se dio gracias al supuesto índice de satisfacción para con sus demandantes o futuros alumnos, y al considerar prioritarias también las necesidades de la planta docente y la generación efectiva de soluciones a problemas en constante crecimiento.

Un caso de importancia trascendental se da en Harvard de Cambridge, Massachusetts, que fue fundada el mes de septiembre de 1636, es decir hace 381 años, siendo la primera de su tipo en la Unión Americana. Según sus enlaces oficiales guarda una tradición ejemplar y gradúa a hombres y mujeres que posteriormente se vuelven personalidades de la política, administración o incluso del espectáculo (Harvard, U. 2018).

Para solicitar el ingreso a sus programas educativos asisten jóvenes de los 50 estados de Norteamérica y de hasta de 80 países de la tierra. En el año 2016 fue nombrada como "la universidad con la mejor reputación en el mundo". Bien, desde su creación, bajo la organización college, se rigió bajo la premisa de contar con docentes que se dedicaran exclusivamente a la docencia. Por algún tiempo, esta responsabilidad la cubrieron algunos egresados, posteriormente se adoptaron los conceptos de docentes y tutores por separado, representada cada área por uno de los maestros ante el consejo.

La semejanza de la efectividad de esta innovación no pasaría desapercibida y es por el año de 1825 cuando Vermont inició actividades dividida por 
departamentos, mientras que 30 años después Wisconsin College siguió el ejemplo; Cornell y Jonh Hopkins establecieron departamentos autónomos (Andaluza, 2010).

Se considera que en Estados Unidos la etapa trascendental del agrupamiento de actividades de acuerdo a funciones o departamentalización fue sobre la década de los 40's, cuando instituciones no relacionadas ofrecieron propuestas diversas que fortalecieron este avance de operación educativa:

-Harvard, permitió inicialmente que fueran los jóvenes quienes prácticamente seleccionaran sus cursos, con el programa curricular abierto.

-Princeton y Columbia asignaron la mitad de la carga curricular y el otro porcentaje similar era dejado a su elección.

-El instituto Superior de Wisconsin, innovó con la modalidad de dividir programas en áreas de mayor y menor demanda de acuerdo a las solicitudes por parte de los escolares.

-Finalmente John Hopkins clasificó a los aspirantes por grupos y especialidad; es decir quienes prefieran las áreas de la salud (enfermería, medicina, odontología etc), al igual que con las Ciencias Humanas y Administrativas. (UACJ, 2006).

\section{La Departamentalización en México}

Como se ha dicho, la iniciativa de trabajo por áreas de especialidad en las instituciones universitarias no es de reciente creación -ya se dijo que desde su inicio la Universidad de Harvard, la más prestigiada de los Estados Unidos, nació con esta práctica hace ya casi 400 años-, mientras que la UACJ trabaja desde el año 2006 con esta herramienta, destacando el experimento de institutos, evidentemente aún falta que se consolide.

Sin embargo otras universidades de México fueron pioneras al experimentar la distribución de las tareas, como es el caso de la Primera Escuela Superior de Guadalajara (U. de G.), la cual ante la cambiante realidad del entorno y de la dinámica social reafirmó su modelo educativo desde una perspectiva flexible que colabora con la coyuntura actual de la realidad, complementado con la estrategia permanente de formación de expertos en reflejo a un eco mecánico de necesidades trascendentales para la comunidad, es decir, planeando a futuro (UAG, 2007).

$\mathrm{Su}$ propuesta le fue llevando a la consecución de un compuesto de pluralidades para equilibrar sus intereses compartidos, así como su proyecto 


\section{DOXA}

pISSN: 2395-8758

eISSN: en trámite

de identidad universitaria, desarrollo cultural y generación del conocimiento para beneficio no sólo de su comunidad sino de su estado y país.

Aunque ha parecido fácil, la experiencia fue emprendida desde el año de 1994 y debió enfrentar los prejuicios, cuestionamientos y escepticismos de una gran parte de su propia población; al igual que otras instituciones, algunas prestigiosas, otras no tanto, pero que la mayoría de ellas se han mostrado reacias a hacer un examen de conciencia y practicar para su propio beneficio, un pertinente análisis sobre sus Modelos Académicos y estructura financiera y docente entre otras.

En la República Mexicana existen 3 mil 324 instituciones de educación superior, entre universidades estatales, privadas, institutos universitarios, escuelas superiores y normales públicas, universidades e institutos tecnológicos y politécnicos universidades estatales con apoyo solidario, incluso las abiertas y a distancia (UNAM, 2016), -aunque algunas fuentes no oficiales manejan el número superior a las 5 mil-.

De ese número ocupan los primeros puestos en el Ranking de las Mejores Universidades del País, la UNAM, encabezando la lista, seguida por la Iberoamericana, el ITESM, la Autónoma Metropolitana (UAM) - fundada el año de 1973 que presumiblemente por su funcionamiento y desarrollo es el ejemplo con más éxito sobre el reagrupamiento según la función-, la de Guadalajara (U. de G.) y la Escuela Nacional de Estudios Profesionales (ENEP).

Coincide que las universidades influyentes en México, también fueron las que nacieron departamentalizadas o pioneras en cambiar el formato Napoleónico al Departamental; aunque no está en la lista de honor, la Autónoma de Ciudad Juárez (UACJ) también trabaja bajo ese régimen (ANUIES, 2017).

La aspiración de la UACH podría ser entonces la manufactura de hombres y mujeres preparados y comprometidos, con alto sentido social: figuras transformadas y transformadoras.

Asimismo podría comprometerse a diseñar y aplicar una estrategia administrativa que detone para beneficio de los futuros profesionistas, egresados, Maestrantes y todo el personal sin importar el nivel dentro del escalafón; con potencial en infraestructura, docentes e investigadores disponibles y todos los recursos con que cuenta, y ciertamente funcionan, pero que podrían ser maximizados. 
Años después, el bosquejo norteamericano fue duplicado en ciudad Juárez por parte de la universidad local (UACJ), ante la obligación que se presentó para satisfacer la demanda de los programas y el acelerado crecimiento de la matrícula.

Se creó en 2006 la llamada Subdirección de Administración de Instalaciones dependiente de la Dirección General de Servicios Administrativos, subdividida en cinco coordinaciones de apoyo a muchas instalaciones, incluyendo las que estaban en el "limbo" administrativo como eran las deportivas y culturales.

El organigrama de la UACJ distribuye mejo el desarrollo de las actividades y en cada subdivisión se puede dar solución, tanto a docentes como a estudiantes, sobre sus demandas y necesidades, aún y cuando existe una autoridad absoluta, todos los institutos tienen autonomía para realizar trámites y tomar decisiones (UACJ, 2015).

En el ejemplo que se muestra, se puede ver que su diseño es demasiado extenso, por lo que es muy factible asumir que la ejecución de este modelo, no representa una disminución de personal, más bien un reajuste de actividades que hacen más eficiente la atención y la administracón de muchas áreas que el Rector por si solo no podría abarcar.

\section{El Proceso Inicial para la Posible Aplicación del Modelo Matricial.}

No es posible definir con total certeza si los cambios que dieron resultado en una institución educativa funcionarán de manera similar en otra. Al trasladar su modelo de trabajo al tipo matricial, por ejemplo, en caso de que la UACH decida replicar -de acuerdo a la infraestructura y expectativas- la fórmula de la UACJ, se tendría que agrupar de acuerdo al área de estudio, los programas de licenciatura.

Por ejemplo, aquellos que abarcan las Ciencias Sociales y Humanidades serían incorporados a un solo control conjunto al lado de Derecho, Economía Internacional, Contaduría y Administración y Ciencias Políticas.

Lo mismo pasaría con Artes, Filosofía y Letras, en tanto que el área de salud agruparía Medicina junto a Odontología, Enfermería y Nutriología e incluso Ciencias de la Cultura Física. Ingeniería, Zootecnia y Ecología, Ciencias Agrícolas y Forestales, al igual que Ciencias Agrotecnológicas formarían otro 


\section{DOXA}

pISSN: 2395-8758

eISSN: en trámite

instituto.

Dicho control de los programas estaría a cargo de un coordinador general, pero se desconoce si fungirá con el cargo de Director; mientras que el Consejo Universitario estaría representado por una persona de cada instituto, muy parecido a su funcionamiento actual, así como los representantes de los empleados académicos.

Sin embargo subsiste la dificultad, además de adecuarse a trabajar sobre un nuevo esquema en lugar del modelo vetusto desarrollado hasta ahora, la estructura de la Autónoma de Chihuahua es inmensa.

El trabajo es extenso y la posterior decisión de lo que habrá de hacerse, implica gran importancia, pues deben estudiarse todas las áreas: desde la distribución que rodea el puesto de la Rectoría y la Secretaría General, así como las Direcciones Académica, Administrativa, de Investigación y Posgrado, Planeación y las de Extensión y Difusión Cultural - áreas que no dependen directamente de esas autoridades.

Presuntamente se reduciría de 15 a solamente 4 el número de titulares de Unidad Académica, (Director); efecto que se daría en cadena bajando a las secretarías que en la actualidad funcionan del acuerdo al Modelo Napoleónico, con la misma conformación, es decir la distribución es como la de Rectoría, pero a escala local.

Juega un papel importante también la reasignación de tareas en lo que ahora son las Secretarías, Coordinaciones, así como el personal administrativo y de talleres, gimnasios, museos, estalaciones deportivas y todo lo que se incluye como infraestructura y personal.

\section{Los Retos Rumbo a la Departamentalización.}

La Universidad Autónoma de Chihuahua vio su nacimiento en el año de 1835 bajo el nombre de Instituto Científico Literario con la asistencia de 22 discípulos que estudiaban latín y gramática; con el paso de los años y el aumento de la demanda, para 1954 el Congreso del Estado determinó su fundación por decreto No. 171 (UACH, 2008).

Ahora el plantel de Educación Universitaria más importante de Chihuahua tiene una extensión de 25 hectáreas en uno de los Campus y otras 200 al norte de la ciudad de la capital, donde crecieron significativamente las instalaciones. 
pISSN: 2395-8758

eISSN: en trámite

DOXA

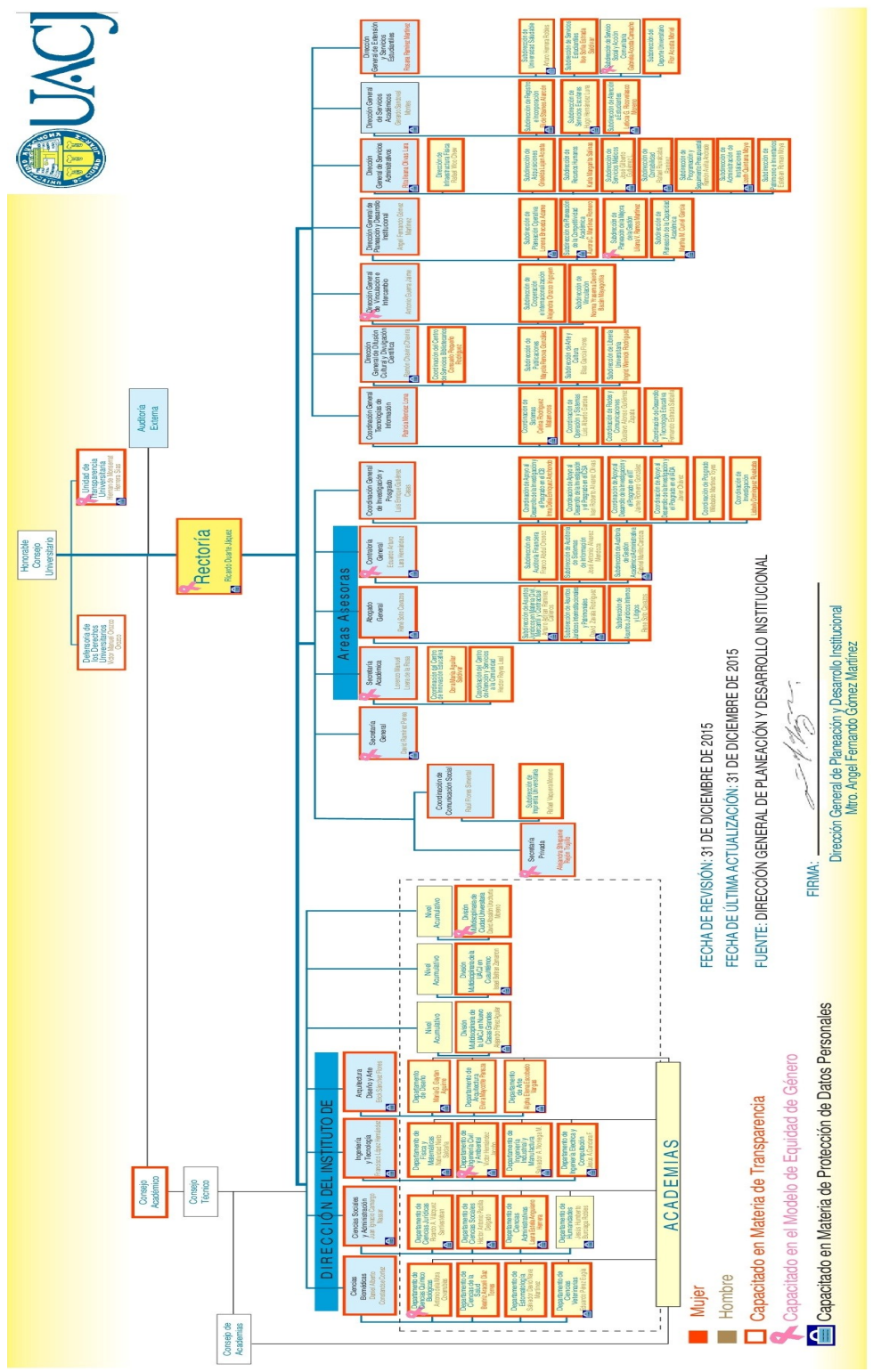

Vol. 8 Núm. 14 Enero - Junio 2018 


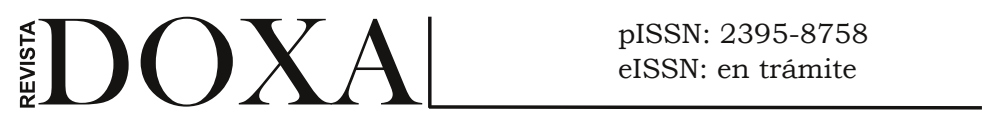

(UACJ, Organigrama de Rectoría UACJ, 2015).
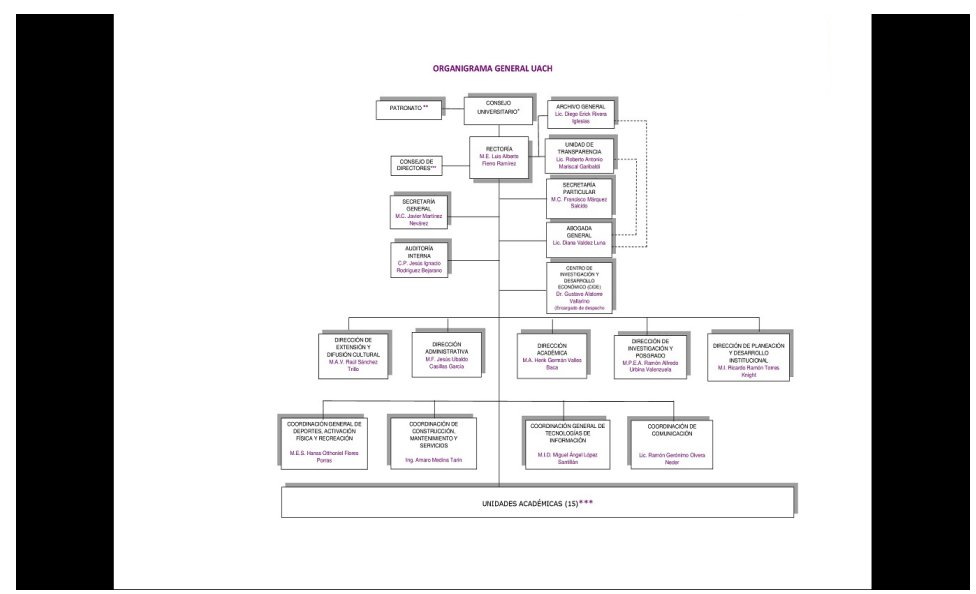

Fuente: Organigrama General UACH. Obtenido de

http://transparencia.uach.mx/articulo_77/fraccion_ii/organigrama_uach_2017_mayo.pdf

Presta atención a un aproximado de 30 mil alumnos con los 127 programas que ofrecen las 15 Facultades por todo el estado y un exitoso programa de Educación Abierta y a distancia que cursan ciudadanos mexicanos que viven dentro y fuera del país, incluyendo Estados Unidos, España y China. Hay quienes aún se mantienen inquietos y escépticos por la iniciativa del recién electo Rector para implementar el Modelo Matricial, pues se dice que anteriormente se intentó promover este programa de mejoramiento a través de los Rectores Dr. Ignacio González Estavillo, Lic. Felipe Lugo Fernández, Dr. Luis Raúl Flores Sánchez, Dr. José Fuentes Mares, Lic. Manuel E. Russek Gameros y los expertos en educación Dr. Pablo Latapí Sarre y Profr. Olac Fuentes Molinar.

El mismo titular actual señaló que el año 1998 se registró ante la Secretaría de Educación Pública (SEP), la Operación Matricial, sin embargo, aparentemente por un error del organismo encargado no se hizo la notificación, de manera que se siguió operando bajo el Modelo Napoleónico. A pesar de ello Fierro Ramírez insiste que ya se dan los primeros pasos para 
pISSN: 2395-8758

eISSN: en trámite

analizar y recomendar todo lo que sea significativo para mejorar, de la misma forma en que hace el llamado a integrar esfuerzos para afrontar la modernidad y competencia; para lo cual se analizan prioridades, tales como:

Inicialmente, gestionar y superar las fronteras institucionales para que se pueda autorizar una reforma a la Ley Orgánica y así libremente promover el cambio del Modelo Napoleónico al Matricial precedido de un exhaustivo análisis como él mismo ha sostenido.

Buscar fortalecer las funciones de investigación para convertir esta parte en un mecanismo de transformación y fortalecimiento de la legitimidad académica, es decir, aumentar la ciencia, separándola de la enseñanza, pero esperando que los resultados sirvan para complementar la docencia; así no se dejará de lado el hecho de que debe existir una vinculación eficiente entre ambas de manera independiente.

Establecer la independencia de los institutos o facultades, docentes, investigadores, carreras y proyectos de investigación social y fortalecer la vinculación con las necesidades del entorno. Acreditación de los programas educativos y acelerar la reforma curricular para fortalecer la preparación superior de los estudiantes.

Igualmente importante es el desarrollo de redes universitarias con otros centros de aprendizaje, empresas, organizaciones sociales y esferas de gobierno con presencia local, regional, estatal, y nacional - buscando dar certidumbre a los futuros profesionistas acerca de las oportunidades que podrían obtener fuera de las aulas -.

Poniendo a su alcance las herramientas necesarias para ser competitivos con egresados de otras instituciones, bajo la premisa de que el demandante mundo laboral cada vez exige mejores especialistas, investigadores y ciudadanos -hombres y mujeres- formados en valores y con un alto sentido de responsabilidad.

Sería injusto no destacar los esfuerzos sustantivos para avanzar dentro de la $\mathrm{UACH}$, circundando la posibilidad de abandonar las premisas del prototipo Napoleónico, pero sin decidir a dar el salto de riesgo, y esperar qué resulta.

Se dio en los últimos años un aumento en la adhesión de Docentes al Perfil PRODEP, también se cuenta con un mayor número de Docentes con grado de Doctor, se incrementó también la incorporación de 123 profesores al Sistema Nacional de Investigación (SNI).

Recientemente se presentó un renovado y eficiente sistema de tutorías, existe 
la permanente capacitación al personal docente y más.

A pesar de todo, persiste la multiplicidad de funciones para los Maestros de Tiempo Completo, ya que siguen desarrollando trabajo fijo como docentes, en la medida de su competencia - pues no hay muchos grupos disciplinares ni cuerpos académicos-. Deben atender lo relacionado a la investigación, la tutoría y hasta la programación deportiva de eventos al mismo tiempo.

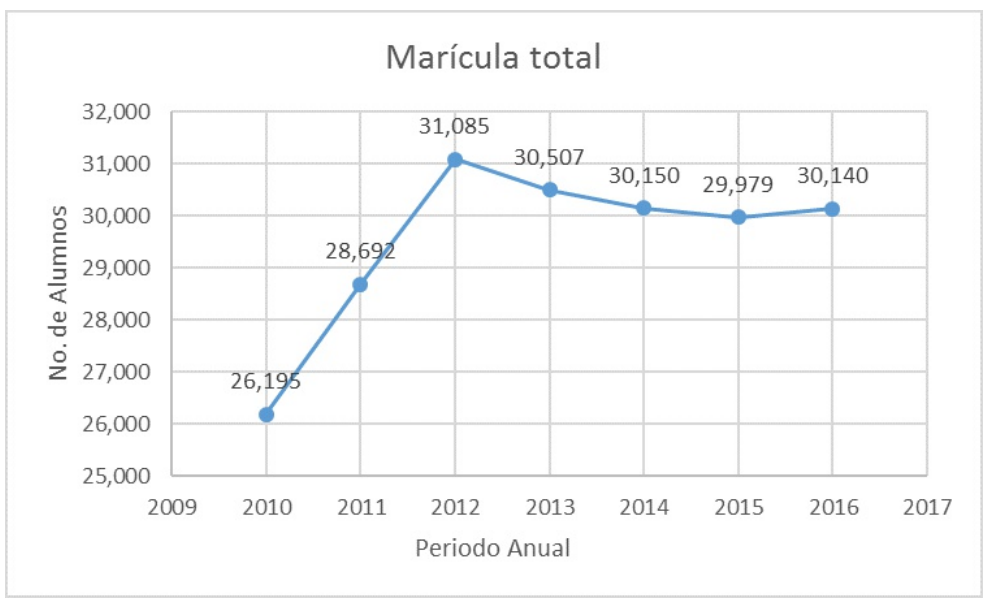

Fuente: Estadística Básica UACH, periodo 2010-2016.

Faltan cosas por hacer, mismas que sin duda ya se encuentran bajo estudio por los especialistas que apoyan el proyecto del titular de la institución; y que, de darse la coyuntura, representarán oportunidades para mejorar la cada vez más eficiente administración de la Universidad Autónoma de Chihuahua.

Tomará tiempo y se verá si posterior al análisis encabezado por Fierro Ramírez, es factible iniciar la traslación aún en su tiempo o quedará a decisión del sucesor en el puesto. Si prevalece la idea de cambio para mejorar y ser más eficientes o se dejan al olvido el trabajo y las propuestas de un sinnúmero de personas interesadas en escalar peldaños hacia la consolidación administrativa, que al tiempo posicione a la institución en mejores niveles de eficiencia.

Hasta ahora, la fijación de las administraciones ha sido sobre todo que se mantengan la demanda de los programas, así como la permanencia de la 
matrícula total; al igual que el aumento en los índices de egresados y titulados. Esto ciertamente aumenta los indicadores, pero aún se requiere avanzar y definir labores u obligaciones sobre áreas específicas como las planteadas anteriormente para concretar el proyecto institucional de la administración actual o la que la sustituya.

\section{Percepción Positiva o Resistencia al Cambio}

La globalización abarca todos los campos. Quienes no se encuentran involucrados en el proyecto de reestructuración administrativa de una institución de nivel superior, posiblemente de manera involuntaria o intencional, adopten posturas tradicionales de resistencia a los métodos de mejoramiento administrativo que se proponen, considerando que el modelo con el cual se trabaja ha dado resultados hasta ahora.

Sin embargo, otras personas con más visión entienden que la evolución tecnológica por la que atravesamos exige renovarse o de lo contrario el desarrollo educativo que se planea únicamente para que dure el periodo de la administración y tenga su final con la misma, en algún momento dejará estancada a la institución entre los paradigmas tradicionales de la educación, es decir se seguirán "acreditando" personas probablemente competitivas que deberán esperar hasta que la demanda de sus capacidades sean requeridas.

A ello se deberá agregar que, a pesar de los esfuerzos parciales por mejorar las condiciones laborales de los docentes, no se ha podido definir cuál será su desempeño o especialidad, y seguirán como tutores, docentes, investigadores, administradores, y funcionarios universitarios e incluso más si es requerido.

Si por otro lado se decide emprender la aventura de examinar el componente total y absoluto del funcionamiento de la universidad, entendiendo que un ejercicio de planeación y ejecución no infalible, encaminado al mejoramiento mediante el reajuste de personal y responsabilidades, no como una estrategia para reducir la nómina y los gastos. Más bien, la oportunidad se presenta a manera de lograr ser eficientes en la operación y dinamizar todas las áreas que integran este Liceo.

\section{Conclusiones}

La idea ya está en camino, serán tiempos de espera e incertidumbre, pero es 


\section{DOXA}

pISSN: $2395-8758$

eISSN: en trámite

posible que, al plantearse adecuadamente este proyecto, posibilite comprobar que puede hacerse efectivo un reordenamiento administrativo que impacte de forma positiva toda la estructura universitaria y dejará constancia de que se puede operar recurso limitado equitativamente distribuido.

Respondiendo a la incertidumbre generalizada: no es necesario tanto personal en algunas áreas, pero no implica que se tenga que disminuir la planta laboral, más bien reorganizar cada estructura y definir el desempeño de cada encargado o auxiliar de área, a fin de evitar la duplicidad de funciones que, en ese caso, volvería a burocratizar el sistema.

Finalmente, el progreso no sólo llegará a las áreas administrativa, sino que servirá para mejorar el desempeño académico, promoción y difusión de la cultura; mejora curricular que podrá empatar la preparación de los egresados con la problemática social, de igual manera tendrán las herramientas para ofrecer soluciones reales a problemas permanentes.

En sí dejará constancia de que en un momento de la historia, la UACH se transformó dejando como legado una Universidad fuerte, integral y que actualmente tiene en las aulas a los elementos que se convertirán en los expertos en distintas áreas.

Serán apoyados en una sólida planta docente, de investigación y promoción cultural, para volverse referente para aquellas entidades que requieran propuestas factibles de solución a la problemática social actual con la garantía de que su trabajo siempre estará respaldado por el absoluto respeto a la dignidad humana y a los valores universales.

\section{Referencias}

Andaluza, E. d. (11 de febrero de 2010). Estados Unidos: un repaso a su historia universitaria. Diario de Sevilla, págs. 04-06. ANUIES. (28 de 03 de 2017). Anuario Estadístico. D. F., México.

Benner, D. (1990). La teoría de la educación de Wilhelm von Humboldt: estudio histórico orientado hacia los problemas de sus relaciones con la reforma pedagógica contemporánea. Munich, Alemania: Ed. 1949.

Colman, E. (2000). Analisis del concepto La justicia de Ulpiano. Revista de Estudiantes de Derecho de la Universidad MOnteavila, 319-326. 
pISSN: 2395-8758

eISSN: en trámite

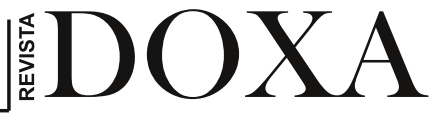

Fierro, L. A. (17 de octubre de 2017). Renovación de la UACH más que una departamentalización: Luis Fierro. Obtenido de http://puentelibre.mx/noticia/100651-

departamentalizacion_uach_luis_alberto_fierro_ramirez_primer_ informe $/ 2$

Harvard, U. (28 de marzo de 2018). Harvard University. Obtenido de https://www.harvard.edu/

Ibarra, A. M. (1998). La Departamentalización, Contexto y Concepto. Revista Electrónica Sinéctica, 1-15.

Jovellanos, M. d. (2017). Estética de la recepción e historia de las ideas en el siglo XVIII. La virtus y el honestum ciceronianos en el honor nobiliario de Montesquieu y la educación cívica. Tópicos, Revista de

Filosofía,

325-372.

Leitner, U. (2008). Los Diarios de Alexander von Humboldt; un mosaico de su conocimineto científico. En M. Cuesta, \& S. Rebok, Alexander Von Humboldt (pág. 387). Madrid, España: Catálogo General de Publicaciones

Oficiales.

Ley Órganica de la UACH, DECRETO No. 953/07 II P.O. (H. Congreso del Estado de Chihuahua 19 de marzo de 2008).

Muñoz, M. A. (viernes de mayo de 2017). La departamentalizacion de la UACh, ¿una opción o una necesidad? El Diario, págs. 10-12 A.

RAE. (2014). Asociación de Academias de la Lengua Española. Madrid. España: Asociación de Academias de la Lengua Española.

Fierro R, L. A. (11 de septiembre de 2017). Luis Fierro explica el proceso de departamentalización. (J. F. Serna, Entrevistador)

Fierro R, L. A. (17 de octubre de 2017). Renovación de la UACH más que una departamentalización: Luis Fierro. Obtenido de http://puentelibre.mx/noticia/100651-

departamentalizacion_uach_luis_alberto_fierro_ramirez_primer_ informe/2

Rodríguez, P. C. (2006). El humanismo en el proyecto educativo de Wilhelm von Humboldt. Juárez, Chihuahua: UACJ.

Rondón, R., \& Perozo, D. (2003). Dos realidades en la Universidad de Zulia y una misma dinámica. Formación Gerencial, 68-87.

Rondón, R., \& Perozo, D. (2003). El Modelo Napoleónico y Matricial. Formación

Gerencial, $69-90$

Vol. 8 Núm. 14 Enero - Junio 2018 


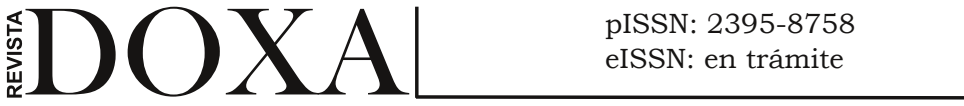

Tisnado, J. R. (noviembre de 2013). Tesis para Obtener el Título Profesional de Licenciado en Administración. Mejoramiento de los Procesos Administrativos de la UGEL No 1 El Porvenir que Contribuya al Desarrollo Educativo de su Jurisdicción en el año 2013. Trujillo,

Provincia Constitucional del Callao, Perú: Repositorio OPAO.

Tünnermann, C. (2003). La Universidad Latinoamericana ante los Retos del Siglo XXI. D.F. México: Unión de Universidades de América Latina, A $\mathrm{C}$

UACH. (07 de marzo de 2008). Historia. Obtenido de http://www.uach.mx/institucional_y_juridica/organizacion/2008 /03/07/historia/

UACJ. (2006). Proceso de Reorganizar de la Gestión Universitaria . Juárez, Chihuahua: Subdirección de Administración de Instalaciones. UACJ. (20 de octubre de 2015). Organigrama de Rectoría UACJ. Obtenido de

http://www.uacj.mx/Transparencia/Documents/Principal/infor macion\%20publica/Trimestre\%20OctubreDiciembre\%202015/Fracci\%C3\%B3n\%20I/Organigrama\%2031\% 20de\%20diciembre\%20de\%202015.pdf

UAG. (2007). Modelo Educativo Siglo 21. Guadalajara, Jalisco: Rectoría General, UAG.

UNAM. (28 de 03 de 2016). Execum Base de Datos. Obtenido de Estudio Comparativo de Universidades Mexicanas: http://www.execum.unam.mx/

Harvard U. (28 de marzo de 2018). Harvard University. Obtenido de https://www.harvard.edu/

Vázquez, M. A. (enero de 2016). Emprendimiento y Universidad Emprendedora. Tesis Doctoral. Valencia, España: Departamento de Proyectos de Ingeniería,Iniversitat Politécnica de Valéncia. 